\title{
Visualization of the Invisible (Editorial)
}

\author{
Terje Midtb $\varnothing^{1}\left(\right.$ D $\cdot$ Lars Harrie $^{2}$
}

Accepted: 6 April 2021 / Published online: 29 April 2021

(C) The Author(s), under exclusive licence to Springer Nature Switzerland AG 2021

\begin{abstract}
Visualizing geographical phenomena often involve communication of information and relationships through a map in either 2D or 3D. In many cases, the information conveyed through the map is a simplified and symbolic depiction of phenomena that is visible in our physical environment. However, maps are also effective for the communication of geographical phenomena that are hidden or are by nature "invisible" for the human eye. As well as invisible factors in our present environment, tracks of occurrences and events from the past have often been lost for many years. In the same way, future situations have still not materialized. This issue covers all these situations. From historical landscapes and events in the past, cadastre and underground geology today and urban planning for the future. The use of traditional cartographic techniques as well as virtual reality are discussed. Several of the research projects involve user studies. The main objectives contributing papers are to make the "invisible" information accessible and more understandable for humans.
\end{abstract}

Keywords Visualization $\cdot$ Thematic map $\cdot$ Make visible $\cdot$ Cartography $\cdot$ User studies

The theme of this issue is the visualization of "invisible" geographic phenomena. However, which phenomena that are visible are not obvious. Our physical environment, including forests, roads, waterbodies, buildings etc. are clearly visible objects which may be included in general maps. On the other hand, the geology in the same area might be only partly visible. While most of the surficial deposits and bedrocks are hidden for the human eye, the top layer, together with the landscape features, can tell us something about the geology below the surface (Harvey 2012). Further, situations and environments in the past, as well as in the future, are not visible for the human eye but can be represented in maps. For example, can landform and quaternary sediments be used to map the coverage and movement of glaciers in the glacial period (Fredin et al. 2013).

Terje Midtbø

terjem@ntnu.no

Lars Harrie

lars.harrie@nateko.lu.se

1 Geomatics group at Department of Civil and Environmental Engineering, Norwegian University of Science and Technology, Høgskoleringen 7, 7048 Trondheim, Norway

2 Department of Physical Geography and Ecosystems Science, Lund University, Sölvegatan 12, SE-223 62 Lund, Sweden
Mapping the invisible is of course nothing new, even though there is a need for improvements in the theory, methods and tools as illustrated in this special issue. One example of visualization of the invisible is linked to the sustainable development goals (SDGs) which were established by the United Nations (UN SDGs 2015). The 17 goals have several things in common, for example that they have a spatial component. Further, several of the 17 themes are not directly visible in our environments. Consequently, when visualizing these goals, thematic maps are excellent for communicating the information. This is demonstrated in the poster collection established by the International Cartographic Association (ICA 2016). This focuses on maps used in connection with each of the SDGs. The poster exhibition is subsequently succeeded by a book which illustrates how cartographic methods can be employed for objective visualization of the invisible (Kraak et al. 2020).

There are many phenomena which by themselves are invisible, while the consequence of the existence of the phenomena is perceivable by humans. As an example, $\mathrm{CO}_{2}$ in the atmosphere is not visible, while extreme weather caused by increasing $\mathrm{CO}_{2}$ concentrations can be perceived by humans in several ways. Neither of the weather phenomena wind nor temperature are directly visible. But there are often distinct visual impacts of both. In a way, we can call them semi-visual. One of the most famous visualizations through other indicators is John Snow's Cholera map from 1854 (Brody et al. 
2000; Field 2020; Snow 1854). The visualization of deaths caused by the epidemic also indicated the origin of the invisible bacteria. Noise and radiation are other examples on invisibility in our environments. Noise is clearly perceivable by humans, but not through vision, while the only way to know about radiation is through sensors and measurements. Various kinds of air pollution are also measured by instruments, though some kinds of air pollution become visible when the concentration is high. In Mohamed et al. (2016), air pollution is measured and visualized by using lichens as indicators.

Short time before the deadline for contribution of research to this special issue, an all-important semi-invisible phenomenon showed up: the COVID-19 pandemic. While the corona virus itself is invisible (for the human eye), the pandemic is more tangible. However, there is a duality between the virus and the disease. Even if the single cases of COVID-19 are easy to comprehend, it is harder to "see" the distribution of the pandemic on a general view, without visualizations by use of cartographic methods. During the last year, we have been introduced to a vast number of thematic maps, charts, graphs etc. to keep us updated about the development of the pandemic. Mocnik et al. (2020) are exploring some challenges and how to approach these challenges when it comes to cartographic visualization of the pandemic.

Another example of invisible information are property boundaries and easements in cadastres. The definition of properties often exists as coordinates and text description in an archive and/or physical boundary markers. These are often difficult to identify in the physical world. Consequently, the legal definition of a property unit is difficult to identify in the real world, even if fences, hedges etc. are used for claiming ownership. This problem is enhanced in 3D cadastres, where property boundaries often coincide with physical structures (walls, ceilings, etc.). At the same time, there are no physical markings of which physical structures that are used. In this issue, Seipel et al. (2020) address the need for proper visualization of 3D cadastre, including easements. They create a visualization system for a $3 \mathrm{D}$ cadastre information, using a Swedish building as a test case. Typical challenges are how to make $3 \mathrm{D}$ visualizations of a $2 \mathrm{D}$ property unit (which legally has no limitations in height) together with 3D property units. Another main challenge is the selection of colours to represent the property boundaries, easements, and physical building structures. To make a useful visualization, transparencies need to be used. This again implies a risk for misinterpretations when colours are overlaid. The similarity of the colours is determined by a colour similarity index that includes hue, saturation and lightness. In their research, Seipel et al. (2020) perform a user study of their visualization system. The result shows that objects, with quite distinct colours when being rendered with no transparency, tend to become increasingly similar as transparency is increased. Generally, the paper by Seipel et al. is very useful for 3D visualization when several feature types are involved, and when there, at the same time, is a need to look through objects (such as walls). In that sense, their result is also applicable outside the 3D cadastre domain, as well as outside the geospatial domain.

Another example of visualization of the invisible in this special issue is within the field of geology. Bang-Kittilsen and Midtbø (2021) study how to visualize uncertainty in geological mapping. The open data sharing policies have made the geological information accessible to a broader user group of non-experts, which entails that it is crucial to develop methods for visualizing uncertainty to increase the usability of the geological information. In their study, Bang-Kittilsen and Midtbø created a web-based experiment to analyze whether and how different design choices influence the sense of uncertainty. The test also included questions about the intuitiveness of the uncertainty for various symbols. Two hundred ten participants from different countries completed the experiment, including both experts and non-experts in geology. The results show that design choices have an impact on the participants' assessment of uncertainty. The design choices that proved to be effective are as follows: changing the appearance of borders between geological layers, making the reference information less detailed and changing scale and symbol size. The dashed line was proven to be a solid choice for experts, and an effective symbol for uncertainty overall. Adding uncertainty into cross-sections could be an excellent tool, which would add understanding both for experts and non-experts. The different answers from experts and nonexperts show a potential for more distinctive communication of the elements that requires domain knowledge.

A typical example of visualization of the invisible is the recreation of a historical landscape, either in photographic or symbolic form. In this issue, Mazagol et al. (2021) recreated the landscape that has, as in thousand other examples, been hidden by man-made reservoir dams. Several of these reservoirs are relatively new, which make the hidden landscape to remain in the memory of the local populations. Today, modern technologies can enable local populations to virtually recreate these lost landscapes and make them visible again. Mazagol et al. (2021) show in a case study how 3D digital tools can effectively recreate representations of a landscape using the valley of the Gorges de la Loire in France as an example. In their work, they digitized several feature types of the past landscape (into vector representation) such as roads, streets, paths, ferries, railways, buildings, factories, mills, castles, canals, bridges, and tunnels. In the visualization, they employ an original automatic colorization method of old greyscale aerial photographs, which make the visualization of the $3 \mathrm{D}$ model similar to old photographs. To summarize, Mazagol et al. (2021) show that developing a historical database can provide a virtual copy of history that supports visualization of the lost landscape. 
Another study of visualization of the past was completed by Romano and Hedley (2021). In their study, they combine GIS and interface technologies for supporting historical narratives. Virtual reality (VR) and augmented reality (AR) equipment are examples on technologies that are suitable interfaces for displaying invisible phenomena in a nature-like manner. One objective of their study was to bridge some of the inabilities in current historical GIS (HGIS) tools. Utilizing the new technologies makes it easier to "engage audiences in 'narratives', 'experiences' or 'emotions' of past events and places". In their research, they work with the historical event of the segregation of Japanese Canadians during World War II after the Canadian government implemented the War Measures Act in 1942. From a parallel project, they have access to a lot of material connected to this event, such as spatial data and analyses, recorded interviews, collections of historical documents (property ownership and community records documents, photographs), textual narratives and conventional maps. The material is concentrated on a small area, a neighbourhood in Vancouver, Canada. The authors conclude that by combining GIS, new interface technologies (such as VR and AR) and interaction design methodologies, we can produce hands-on prototypes that allow us to deliver spatial and emotional immersion.

As well as the past is invisible for us, the future is likewise. How to visualize the future is addressed by Judge and Harrie (2020). Their use case is focused on visualization of detailed development plans (DDPs) that legally define what is allowed to be built on a specific property. A proper visualization of these plans is important to facilitate public participation in the urban planning process. In most countries, visualizations of DDPs are still in the form of static 2D maps. However, there is a movement towards 3D interactive maps. This could potentially benefit public participation by improving communication of the plan proposal. But it also raises several challenges. One challenge is that an interactive 3D map can give the impression that this is how the future will look like, while a DDP visualization aims at showing what could be built within the legal frame of the DDP. This implies that the uncertainty of the future needs to be addressed in the cartographic design. In their study, Judge and Harrie developed and implemented preliminary guidelines of a 3D DDP visualization, including the possibilities to explicitly address how to handle uncertainty in DDP visualization by interactive tools. The preliminary guidelines were then evaluated by semi-structured interviews with urban planning professionals. Based on the outcome of these interviews, the guidelines were updated. Since a symbolic representation better reflects the uncertain relationship between a DDP and the (possible) physical realizations of the plan, nature-like presentations are avoided in the final guidelines.

The study by Gupta et al. (2020) also addresses the planning perspective. In their case, this is visualization of spatial and temporal clustering of rural assets created under the world's largest social safety programme, MGNREGA, in the Prakasam district of Andhra Pradesh, India. The study is based on exploratory spatial data analysis (ESDA) to reveal interesting facts about the various environmental socio-economic issues in the MGNREGA program. More specifically Gupta et al. applied hotspot analysis which shows that most of the assets tend to cluster at village level at selected locations and particular periods during the MGNREGA project implementation. The authors conclude that ESDA tools are highly useful for simultaneous visualization of spatial and temporal clusters. The empirical results presented in the study could be helpful and valuable in enhanced planning and implementation of MGNREGA, as well as other similar rural development programs.

The six papers in this special issue nicely exemplify several challenges of visualization of invisible phenomena as well as provide some theories, methods and tools for the visualization. A central question is who the intended users of these tools are. Experts are very clever to read and interpret data in their field of expertise, even if these data are incomprehensible for most people. They clearly could benefit from a tool that shows all the complexity of the information. To communicate the information to the public (and politicians and other decision makers!), it is essential to visualize the data through more structured presentations. The most sensible way to verify if a method/tool is fulfilling its objective (for the intended user group) is to utilize user preference or usability studies. We note that user studies have been widely applied by the authors in this special issue. In Seipel et al. (2020), they perform a user study (of professional users) of their 3D cadaster visualization system; in the study by BangKittilsen and Midtbø (2021), a wide and large test group ( $>200$ persons) performs an evaluation of the design proposals for uncertainty presentations; and finally, in Judge and Harrie (2020), an expert user group is interviewed about the recommended cartographic design of the interactive 3D detail development plan visualizations. This focus of the users is inherently important in a time of fast technical development, nicely illustrated by Romano and Hedley (2021) in this issue, and an increasing availability of expert data for the public (Bang-Kittilsen and Midtbø 2021).

The importance of visualization of the invisible is likely to increase. For example, the technological evolution contributes with a wide-ranging growth of various kinds of sensors, and several of those produce data with a spatial component. As a result of this, the availability and quality of (invisible) geographical related information are steadily increasing. These data can be conveyed as information through both known and new, innovative visualization methods. This special issue did not include any paper explicitly addressing the case of visualization of sensor data. However, we anticipate that there will be a growing interest in visualizing of this kind of data.

Acknowledgements We would like to thank all authors for their excellent contributions, as well as the reviewers for their valuable advice to ensure the scientific quality of the individual contributions. We would also like 
to thank the editors-in-chiefs, especially Haowen Yan for his support and contribution when preparing this special issue.

\section{Declarations}

All accepted principles of ethical and professional conduct have been followed during this research in accordance with Springer's standards.

Ethical Approval This manuscript has not been published, accepted for publication, or is under editorial review for publication elsewhere.

Conflict of Interest The authors declare no competing interests.

\section{References}

Bang-Kittilsen A, Midtbø T (2021) Imaging the subsurface: how different visualizations of cross-sections affect the sense of uncertainty. $J$ Geovisual Spat Analys 5(1). https://doi.org/10.1007/s41651-02000071-6

Brody H, Rip MR, Vinten-Johansen P, Paneth N, Rachman S (2000) Map-making and myth-making in Broad Street: the London cholera epidemic. Lancet 356(9223):64-68. https://doi.org/10.1016/S01406736(00)02442-9

Field, K. (2020). Something in the water: the mythology of Snow's map of cholera. ArcGIS Blog. https://www.esri.com/arcgis-blog/ products/arcgis-pro/mapping/something-in-the-water-themythology-of-snows-map-of-cholera/

Fredin O, Bergstrøm B, Eilertsen R, Hansen L, Longva O, Nesje A, Sveian H (2013) Glacial landforms and quaternary landscape development in Norway. Norges Geologiske Undersøkelse, Special Publ 13:5-25

Gupta S, Dharmaraj T, Reddy KM, Ravisankar T (2020) Spatial-temporal analysis and visualization of rural development works implemented under world's largest social safety programme in India - a case study. J Geovisual Spat Analys 4(21). https://doi.org/10.1007/ s41651-020-00062-7
Harvey A (2012) Introducing geomorphology: a guide to landforms and processes. Dunedin Academic Press Ltd. ISBN: 978-1-903544-79-2

ICA. (2016). Maps and sustainable development goals. International Cartographic Association. https://icaci.org/maps-and-sustainabledevelopment-goals/

Judge S, Harrie L (2020) Visualizing a possible future: map guidelines for a 3D detailed development plan. J Geovisual Spat Analys 4(7). https://doi.org/10.1007/s41651-020-00049-4

Kraak, M.-J., Roth, R. E., Ricker, B., Kagawa, A., \& Le Sourd, G. (Eds.). (2020). Mapping for a sustainable world. United Nations together with International Cartographic Association. https://www.un.org/ geospatial/programmes

Mazagol PO, Niogret P, Riquier J, Depeyre M, Ratajczak R, CrispimJunior CF, Tougne L (2021) Tools against oblivion: 3D visualization of sunken landscapes and cultural heritages applied to a dam reservoir in the Gorges de la Loire (France). J Geovisual Spat Analys 5(2). https://doi.org/10.1007/s41651-020-00072-5

Mocnik F-B, Raposo P, Feringa W, Kraak M-J, Köbben B (2020) Epidemics and pandemics in maps - the case of COVID-19. J Maps 16(1):144-152. https://doi.org/10.1080/17445647.2020. 1776646

Mohamed AH, M'hamed M, Hichem MF, B. M. (2016) Air pollution mapping with bio-indicators in urban areas. Air Qual - Measure Model. https://doi.org/10.5772/65299

Romano S, Hedley N (2021) Daylighting past realities: making historical social injustice visible again using HGIS-based virtual and mixed reality experiences. J Geovisual Spat Analys 5(8). https://doi.org/10. 1007/s41651-021-00077-8

Seipel S, Andrée M, Larsson K, Paasch JM, Paulsson J (2020) Visualization of 3D property data and assessment of the impact of rendering attributes. J Geovisual Spat Analys 4(23). https://doi.org/ 10.1007/s41651-020-00063-6

Snow J (1854) The cholera near Goldensquare, and at Deptford. Med Times Gazette 9:321-322

UN SDGs (2015). THE 17 GOALS | Sustainable development. (n.d.). https://sdgs.un.org/goals

Publisher's Note Springer Nature remains neutral with regard to jurisdictional claims in published maps and institutional affiliations. 